\title{
Potential role of incretins in diabetes and COVID-19 infection: a hypothesis worth exploring
}

\author{
Paola Pantanetti ${ }^{1}$ (D . Giovanni Cangelosi ${ }^{1} \cdot$ Giuseppe Ambrosio $^{2}$
}

Received: 18 May 2020 / Accepted: 27 May 2020 / Published online: 26 June 2020

(c) Società Italiana di Medicina Interna (SIMI) 2020

\begin{abstract}
Patients with diabetes mellitus have been reported to be at a high risk of complications from SARS-CoV2 virus infection (COVID-19). In type 2 diabetes, there is a change in immune system cells, which shift from an anti-inflammatory to a predominantly pro-inflammatory pattern. This altered immune profile may induce important clinical consequences, including increased susceptibility to lung infections; and enhanced local inflammatory response. Furthermore, dipeptidyl peptidase 4 (DPP4) enzyme is highly expressed in the lung, and that it may have additional actions besides its effects on glucose metabolism, which might exert profound pro-inflammatory effects. We briefly review the impact on the inflammatory system of DPP4 for its possible detrimental effect on COVID-19 syndrome, and of DPP4 inhibitors (gliptins), currently used as glucose lowering agents, which may have the potential to exert positive pleiotropic effect on inflammatory diseases, in addition to their effects on glucose metabolism. Thanks to these ancillary effects, gliptins could potentially be "repurposed" as salutary drugs against COVID-19 syndrome, even in non-diabetic subjects. Clinical studies should be designed to investigate this possibility.
\end{abstract}

Keywords Diabetes $\cdot$ Dipeptidyl peptidase-4 $\cdot$ COVID-19 $\cdot$ Gliptins $\cdot$ Inflammation

\section{Introduction}

In December 2019, many cases of viral pneumonia were identified in Wuhan, caused by a new strain of virus, coronavirus (SARS-CoV2). In humans, coronaviruses typically cause a mild disease of the upper respiratory tract, but they can also be associated with more serious lung diseases, mainly in subjects with multiple comorbidities. In this respect, SARS-CoV2 infection may cause a more severe condition, known as COVID-19 syndrome. This epidemic quickly spread to a growing number of countries, counting to date hundreds of thousands of cases. COVID-19 has already caused more deaths than previous SARS-CoV and MERS$\mathrm{CoV}$ epidemics combined. Therefore, therapies are urgently needed to help curb COVID-19 rapid spread, and minimize its high mortality rate.

Paola Pantanetti

paola.pantanetti@sanita.marche.it

1 UO Diabetologia Asur Marche, Area Vasta 4, Via Dante Zeppilli 18, 63900 Fermo, Italy

2 Division of Cardiology, School of Medicine, University of Perugia, Perugia, Italy

\section{Diabetes and COVID-19}

Epidemiological analyses have found a strong correlation between presence of pre-existing comorbidities and development of serious diseases following SARS-CoV2 infection [1]. Risk factors associated with development of serious morbidity or deaths are: male gender, advanced age, and number of comorbidities. In particular, diabetes, renal failure, cardiac, respiratory, cerebrovascular disease, and hypertension, have all been shown to predispose patients to more complicated clinical pictures with consequent increase in risk for lethal outcomes [2, 3]. Overlapping risks among diabetic subjects have been described during the two previous COV infections, namely the severe acute respiratory syndrome (SARS) of 2002, which involved more than 8000 individuals mainly in Asia [4], and the Middle East respiratory syndrome (MERS), which in 2012 affected over 2000 people mainly in Saudi Arabia [5]. Data on COVID-19 in patients with diabetes are accumulating. Overall percentage of diabetics affected by COVID-19 has been reported between 5.3\% and 20\% [2]. In intensive care unit (ICU) patients, Yang et al. observed that major comorbidities of COVID-19 patients who did not survive were cerebrovascular disease (22\%) and diabetes (22\%) [6]. A 
report of 72.314 COVID-19 cases by the Chinese Center for Disease Control and Prevention showed an overall mortality of $2.3 \%$, which rose to $7.3 \%$ in diabetics [7]. In Italy (15 May 2020), 223.095 cases with 29.884 deaths were confirmed and more comorbidities were observed in COVID-19 patients who died. The report from the Italian National Institute of Health, indicates that a large number of COVID-19 patients are affected by diabetes (30.5\%) [8].

\section{Diabetes and inflammation in the setting of COVID-19}

Understanding how diabetes contributes to the severity of the disease, and how to treat COVID-19 syndrome, may be of utmost importance. The pathophysiology of this association remains uncertain today. Previous studies have shown that diabetic patients are more susceptible to infections, in particular flu and pneumonia. Indeed, diabetes is not just a disorder of glucose metabolism, but it can also be viewed as a chronic inflammatory state, which is also accompanied by alterations in immune system; in metabolic disorders, altered activity of macrophages and lymphocytes impairs immune function, with development of major complications. In type- 2 diabetes, there is a change in immune system cells residing in adipose tissue, shifting from an anti-inflammatory, macrophages, and regulatory $\mathrm{T}$ cells (Tregs) to a predominantly pro-inflammatory pattern, macrophages, and CD1 + Th1 and Th $17 \mathrm{~T}$ cells, interleukin-1 (IL-1), tumor necrosis factor- $\alpha$ (TNF- $\alpha$ ), which promotes systemic insulin resistance and pancreatic beta-cell injury (Table 1) [9]. This altered immune profile induces many consequences, including increased susceptibility to infections. In patients with viral pneumonia, Guo et al. found that the absolute count levels of $\mathrm{CD} 3+\mathrm{T}$ cells, $\mathrm{CD} 8+\mathrm{T}$ cell.s and CD $3+\mathrm{CD} 4+\mathrm{T}$ cells in patients who died were higher than in the survivors [10].

Table 1 Most important proand anti-inflammatory cytokines

\section{Dipeptidyl peptidase-4 and pulmonary inflammation}

The response to virus-induced tissue damage could result in respiratory failure with acute respiratory distress syndrome (ARDS), characterized by rapid onset of widespread inflammation in the lungs [11]. Numerous studies have shown that pro-inflammatory cytokine levels are extremely high in subjects with severe/critical COVID-19. Cytokine storm syndrome (CSS) seems to be of particular concern in patients with severe or critical conditions of infection. COVID-19 patients have high levels of proinflammatory cytokines in lung; thus, blocking circulating IL-6, IL-1, and TNF could be effective for patients in respiratory insufficiency by COVID-19 [12].

Gut-derived incretins such as glucagon-like peptide-1 (GLP-1) and glucose-dependent insulinotropic peptide (GIP) are necessary for glycemic control. In particular, GLP-1 is secreted after a meal, and it lowers glucose concentrations by increasing insulin secretion and suppressing glucagon release. DPP4 rapidly degrades circulating GLP-1 and GIP, thus contributing to glycemic homeostasis. However, a series of indirect findings suggest that the DPP4 system can have important biological actions beyond glucose metabolism as DPP4 can have a pro-inflammatory action, stimulating the production of IL- 6 and TNF- $\alpha$ in monocytes, as well as exert a possible modulating function in the immune system. This trans-membrane glycoprotein, known also as CD26 lymphocyte cell surface protein, is expressed in different types of immune cells, and it is supposed to influence their function: $\mathrm{CD} 4+, \mathrm{CD} 8+\mathrm{T}$ cells, $B$ cells, NK cells, dendritic cells, and macrophages. In principle, therefore, DPP4 is capable to modulate many cytokines, chemokines, and peptide hormones [13]. As noted, DPP4/CD26 is directly involved in various immune or inflammatory diseases. In the lungs of healthy subjects, DPP4/CD26 is mainly present on type I and II alveolar cells, alveolar macrophages, vascular endothelium, and pleural mesothelium. Previous studies have shown a direct functional role for DPP4/CD26, in pulmonary diseases, such as asthma, chronic obstructive pulmonary disease, and lung fibrosis (Fig. 1).

\begin{tabular}{ll}
\hline Pro-inflammatory & $\begin{array}{l}\text { Anti- } \\
\text { inflam- } \\
\text { matory }\end{array}$ \\
\hline IL-1 $\beta$ & IL-1ra \\
IL-6 & IL-4 \\
IL-8 & IL-6 \\
TNF- $\alpha$ & IL-10 \\
Chemokines & IL-11 \\
Interferons & IL-13 \\
\hline
\end{tabular}

\section{Dipeptidyl peptidase-4 inhibition and pulmonary inflammation}

If DPP4 system is also involved in immunological and inflammatory processes, it would be expected that inhibiting this pathway should translate into beneficial effects in this respect. This possibility has recently been tested, 
Fig. 1 Mechanistic effects of DPP4 inhibition in lung injury ( modified from Shao et al. 2020) [13]

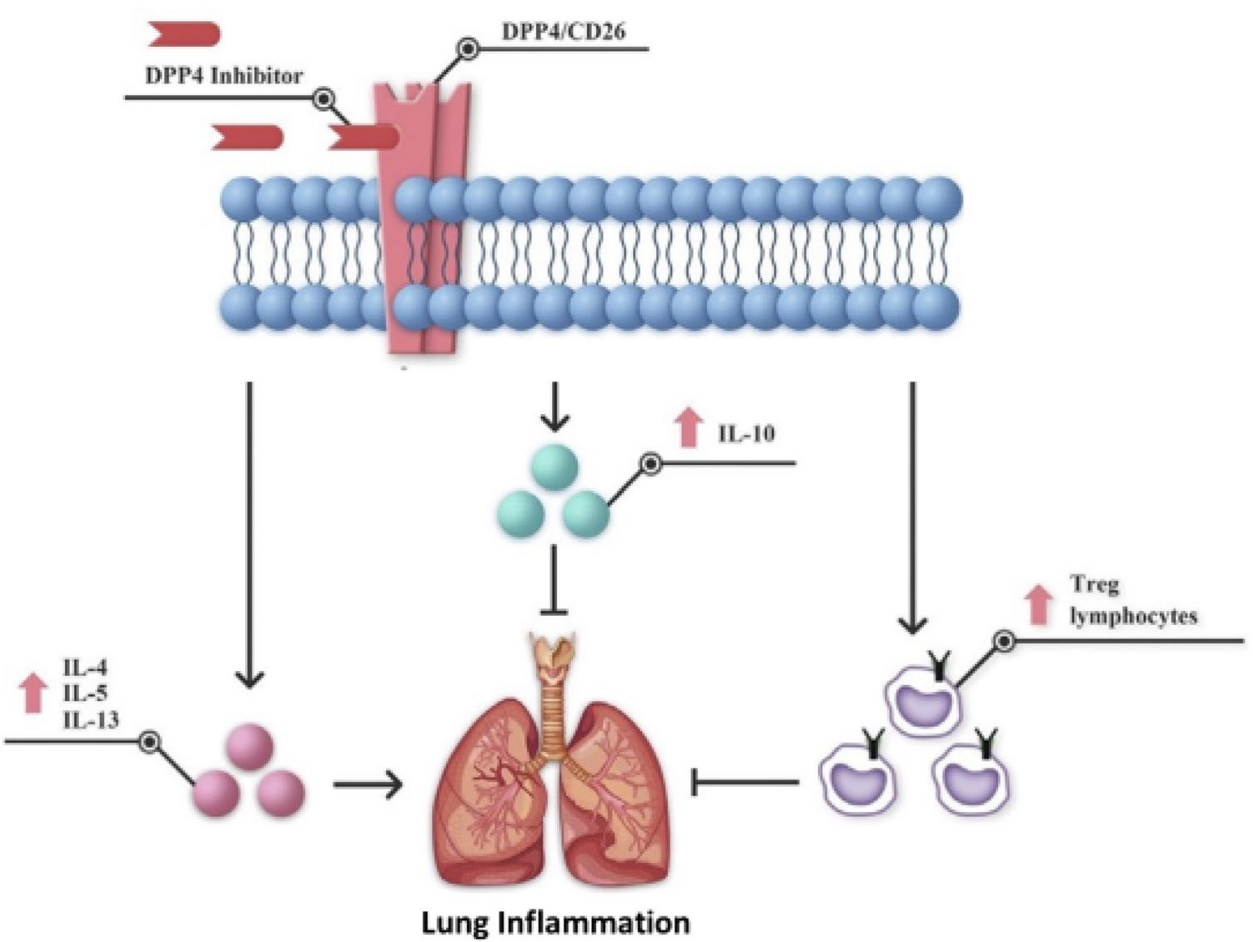

thanks to the availability of specific inhibitors of DPP4 (DPP4is). DPP4is, commonly called gliptins, have been initially developed and are currently widely used as effective oral hypoglycemic drugs in the treatment of type-2 diabetes mellitus (T2DM). However, DPP4 inhibition has the potential to exert a positive pleiotropic effect on vascular and inflammatory diseases [14, 15], or even direct anti-inflammatory effect on various cell types: $\mathrm{T}$ cells, macrophages, and adipocytes [13, 16, 17]. With specific respect to lung diseases, Kawasaki et al. demonstrated the direct anti-inflammatory effects of DPP4 inhibition on LPS-induced lung injury in mice and in human lung epithelial cells. Similarly, DPP4i administration in mice is able to exert protective effects in the lung [17]. Telikani et al. have determined serum levels of TNF- $\alpha$, IL- $1 \beta$, and IL-10 in 60 patients with T2DM; sitagliptin treatment significantly decreased the high levels of pro-inflammatory TNF- $\alpha$, and at the same time increased anti-inflammatory IL-10 concentrations [18]. Recently, Soare et al. have shown that DPP4 promotes activation of fibroblasts, induced by transforming growth factor $\beta$ (TGF- $\beta$ ), which is instrumental to initiate tissue fibrosis: conversely, inactivation of DPP4 exerted important anti-fibrotic effects in several experimental models of pulmonary and skin fibrosis [19]. Recently, the DPP4i, Sitagliptin, has proven to exert protective effect against LPS-induced lung injury in mice. This effect might be mediated through a combination of several mechanisms, including a potent anti-inflammatory action, as demonstrated by a significant reduction of cytokines in the lung.

\section{Dipeptidyl peptidase-4 inhibition and COVID-19}

Collectively, these observations explain the involvement of DPP4/CD26 in the immune system and suggest a possible use of DPP4 inhibitors as immunomodulatory drugs in COVID-19. Indeed, Sadikot et al. have recently proposed that GLP-1 may constitute a novel therapeutic approach to ARDS, as they demonstrated that human GLP-1 inhibits nuclear factor-B (NF-KB) activation in cultured macrophages and in a murine model of acute lung injury [20]. Given the already established safety profile of these classes of drugs, and their clinical availability, they might be "repurposed" as potential therapeutic drug candidates for coronavirus pneumonia in T2DM patients. Interestingly, as gliptins are known to have little or no hypoglycemic effects [14, 15], they might be used safely also in non-diabetic patients.

\section{Conclusions}

There is an urgent need to develop and test novel therapeutic approaches to treat this devastating COVID-19 disorder. Future studies are warranted to evaluate the use of 
gliptins in modulating the inflammatory response of distal respiratory tract, with the aim of improving prognosis and reducing mortality of respiratory infections in patients with COVID-19.

\section{Compliance with ethical standards}

Conflict of interest The authors declare that they have no conflict of interest.

Statements on human and animal rights This article does not contain any studies with human participants or animals performed by any of the authors.

Informed consent None.

\section{References}

1. Farsalinos K, Barbouni A, Niaura R (2020) Systematic review of the prevalence of current smoking among hospitalized COVID-19 patients in China: could nicotine be a therapeutic option? Intern Emerg Med. https://doi.org/10.1007/s11739-020-02355-7

2. Deng SQ, Peng HJ (2020) Characteristics of and public health responses to the coronavirus disease 2019 outbreak in China. J Clin Med. https://doi.org/10.3390/jcm9020575

3. Violi F, Pastori D, Pignatelli P, Cangemi R (2020) SARS-CoV-2 and myocardial injury: a role for Nox2? Intern Emerg Med. https ://doi.org/10.1007/s11739-020-02348-6

4. Wang D, Hu B, Hu C et al (2020) Clinical characteristics of 138 hospitalized patients with 2019 novel coronavirus-infected pneumonia in Wuhan, China. JAMA 323:1061-1069. https://doi. org/10.1001/jama.2020.1585

5. Morra ME, Van Thanh L, Kamel MG et al (2018) Clinical outcomes of current medical approaches for Middle East respiratory syndrome: a systematic review and meta-analysis. Rev Med Virol 28:e1977

6. Yang X, Yu Y, Xu J et al (2020) Clinical course and outcomes of critically ill patients with SARS.CoV-2pneumonia in Wuhan, China: a single-centered, retrospective, observational study. Lancet Respir Med 8:475-481. https://doi.org/10.1016/S2213 $-2600(20) 30079-5$

7. Wu Z, McGoogan JM (2020) Characteristics of and important lessons from the coronavirus disease 2019 (COVID-19) outbreak in China: summary of a report of 72314 cases from the Chinese center for disease control and prevention. J Am Med Assoc. https ://doi.org/10.1001/jama.2020.2648

8. Istituto Superiore della Sanità (ISS) (2020) Characteristics of Covid-19 patients dying in Italy. 15 May 2020. https://www.epice ntro.iss.it/en/coronavirus/bollettino/Report-COVID-2019
9. Meshkani R, Vakili S (2016) Tissue resident macrophages: key players in the pathogenesis of type 2 diabetes and its complications. Clin Chim Acta 462:77-89

10. Guo L, Wei D, Zhang X et al (2019) Clinical features predicting mortality risk in patients with viral pneumonia: the MuLBSTA score. Front Microbiol 10:2752. https://doi.org/10.3389/fmicb .2019 .02752

11. Xu Z, Shi L, Wang Y et al (2020) Pathological findings of COVID-19 associated with acute respiratory distress syndrome. Lancet Respir Med 8:420-422. https://doi.org/10.1016/S2213 -2600(20)30076-X

12. Shi Y, Wang Y, Shao C et al (2020) COVID-19 infection: the perspectives on immune responses. Cell Death Differ. https://doi. org/10.1038/s41418-020-0530-3

13. Shao S, Xu Q, Yu X, Pan R, Chen Y (2020) Dipeptidyl peptidase 4 inhibitors and their potential immune modulatory functions. Pharmacol Ther. https://doi.org/10.1016/j.pharmthera.2020.10750 3

14. Seferovic PM, Coats AJS, Ponikowski P et al (2020) European Society of Cardiology/Heart Failure Association position paper on the role and safety of new glucose-lowering drugs in patients with heart failure. Eur J Heart Fail 22:196-213

15. Cattadori G, Pantanetti P, Ambrosio G (2019) Glucose-lowering drugs and heart failure: implications of recent cardiovascular outcome trials in type 2 diabetes. Diabetes Res Clin Pract 157:107835. https://doi.org/10.1016/j.diabres.2019.107835

16. Reinhold D, Bank U, Bühling F, Kähne T, Kunt D, Faust J, Neubert K, Ansorge S (1994) Inhibitors of dipeptidyl peptidase IV (DP IV, CD26) specifically suppress proliferation and modulate cytokine production of strongly CD26 expressing U937 cells. Immunobiology 192:121-136. https://doi.org/10.1016/S0171 $-2985(11) 80412-2$

17. Kawasaki T, Chen W, Htwe YM, Tatsum K, Dudek SM (2018) Some of the protective effects of sitagliptin could be mediated through extra-glycemic action, as modulating DPP4/CD26 in rats, the DPP4 inhibition of sitagliptin improves LPS-induced lung injury. Am J Physiol Lung Cell Mol Physiol 315:L834-L845. https://doi.org/10.1152/ajplung.00031

18. Telikani Z, Sheikh V, Zamani A et al (2020) Effect of sitagliptin on serum levels of TNF- $\alpha$, IL- $1 \beta$ and IL-10 in patients with type 2 diabetes mellitus. Koomesh 22:71-77

19. Soare A, Györfi HA, Matei AE et al (2020) Dipeptidyl-peptidase-4 as a marker of activated fibroblasts and a potential target for the treatment of fibrosis in Systemic Sclerosis. Arthritis Rheumatol 72:137-149. https://doi.org/10.1002/art.41058

20. Sadikot RT, Rubinstein I (2009) Long-acting, multi-targeted nanomedicine: addressing unmet medical need in acute lung injury. $\mathbf{J}$ Biomed Nanotechnol 5:614-619

Publisher's Note Springer Nature remains neutral with regard to jurisdictional claims in published maps and institutional affiliations. 